\title{
Erratum
}

\section{Utility of delayed surgical repair of neonatal brachial plexus palsy}

\author{
Clinical article
}

To The EDITOR: We thank you for publishing our paper, "Utility of delayed surgical repair of neonatal brachial plexus palsy. Clinical article." (J Neurosurg Pediatr 13:462-470, 2014). After our paper had been published, a Letter to the Editor by Dr. Willem Pondaag and Dr. Martijn J. A. Malessy made us aware of some errors. The errors center around papers we cited in Table 3. We are taking this opportunity to make corrections.

The original Reference 18 in our manuscript (Chuang DC: Nerve transfers in adult brachial plexus injuries: my methods. Hand Clin 21:71-82, 2005) does not accurately reflect the article from which we extracted surgical results. We have added an additional reference, now listed as Reference 18a:

Chuang DC, Mardini S, Ma HS: Surgical strategy for infant obstetrical brachial plexus palsy: experiences at Chang Gung Memorial Hospital. Plast Reconstr Surg 116:132-144, 2005.

The original Lin and Lin reference in Table 3 (Reference 54, Lin CC, Lin J: Brachial plexus palsy caused by secondary fracture displacement in a patient with closed clavicle fracture. Orthopedics 32:769, 2009) should be replaced with Reference 55:

Lin JC, Schwentker-Colizza A, Curtis CG, Clarke HM: Final results of grafting versus neurolysis in obstetrical brachial plexus palsy. Plast Reconstr Surg 123:939-948, 2009.

We are pleased to have the opportunity to correct these errors. The corrected Table 3 is shown below.

Please include this information when citing this paper: published online August 22, 2014; DOI: 10.3171/2014.7.PEDS13382a.
TABLE 3: References used for surgical cases*

\begin{tabular}{|c|c|c|c|c|}
\hline \multirow[b]{2}{*}{ Authors \& Year } & \multirow{2}{*}{$\begin{array}{l}\text { No. of } \\
\text { Cases }\end{array}$} & \multicolumn{3}{|c|}{ Timing of Surgical Repair (pt age) } \\
\hline & & 3 Mos & 6 Mos & 12 Mos \\
\hline Slooff \& Ubachs, 1993 & 17 & & $x$ & \\
\hline Laurent \& Lee, 1994 & 52 & $X$ & & \\
\hline Xu et al., 2000 & 8 & & $X$ & \\
\hline Dumont et al., 2001 & 13 & & $X$ & \\
\hline Kawabata et al., 2001 & 11 & & $x$ & \\
\hline Blaauw \& Sloof, 2003 & 25 & & $x$ & \\
\hline Grossman et al., 2004 & 11 & & & $X$ \\
\hline Birch et al., 2005 & 107 & & $x$ & \\
\hline $\begin{array}{l}\text { Chuang, } 2005 \& \text { Chuang } \\
\text { et al., } 2005\end{array}$ & 16 & & $X$ & \\
\hline Pondaag et al., 2005 & 69 & & $X$ & \\
\hline O’Brien et al., 2006 & 37 & & $x$ & \\
\hline Ashley et al., 2007 & 47 & & $x$ & \\
\hline Kirjavainen et al., 2007 & 85 & $X$ & & \\
\hline Pondaag \& Gilbert, 2008 & 20 & & $X$ & \\
\hline Lin et al., 2009 & 56 & & & $X$ \\
\hline Wellons et al., 2009 & 20 & & $X$ & \\
\hline Yam et al., 2009 & 13 & & $x$ & \\
\hline
\end{tabular}

${ }^{*} \mathrm{pt}=$ patient.

We apologize to the editor and readers. The errors have been corrected online as of August 22, 2014.

ZARINA S. Ali, M.D. ${ }^{1}$ ERIC L. ZAGER, M.D. ${ }^{1}$ Gregory G. Heuer, M.D., Ph.D. ${ }^{1,2}$ Sherman C. Stein, M.D. ${ }^{1}$ ${ }^{1}$ University of Pennsylvania Philadelphia, PA

${ }^{2}$ The Children's Hospital of Philadelphia Philadelphia, PA 